Document downloaded from:

http://hdl.handle.net/10251/147547

This paper must be cited as:

Ferreyra, DE.; Levis, F.; Thome, N. (2018). Characterizations of k-commutative equalities for some outer generalized inverses. Linear and Multilinear Algebra. 1-16. https://doi.org/10.1080/03081087.2018.1500994

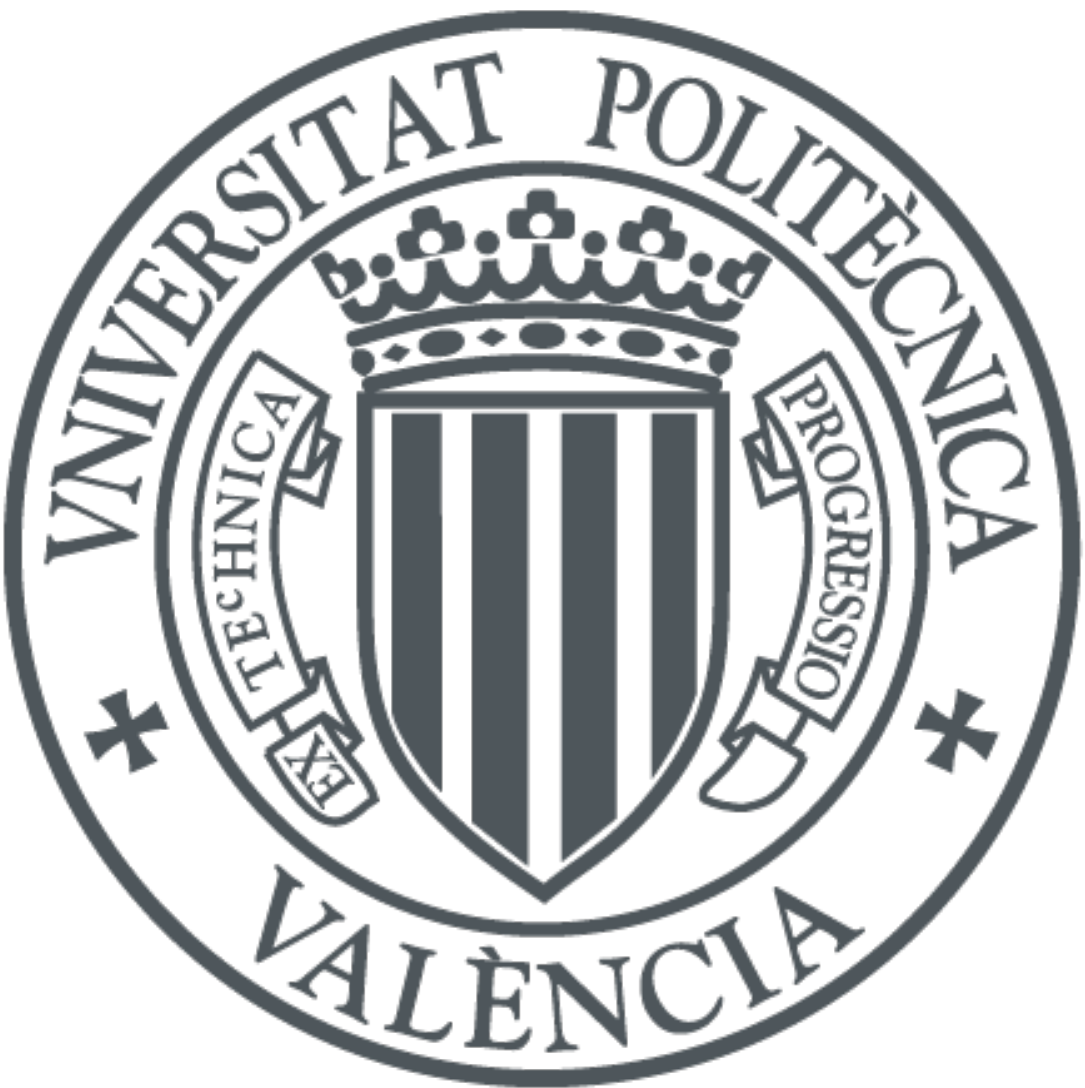

The final publication is available at

https://doi.org/10.1080/03081087.2018.1500994

Copyright Taylor \& Francis

Additional Information 


\title{
Characterizations of $k$-commutative equalities for some outer generalized inverses
}

\author{
D.E. Ferreyra*, F.E. Levis* ${ }^{*}$ N. Thome ${ }^{\dagger}$
}

\begin{abstract}
In this paper, we present necessary and sufficient conditions for the $k$-commutative equality $A^{k} X=X A^{k}$, where $X$ is an outer generalized inverse of the square matrix $A$. Also, we give new representations for core EP, DMP, and CMP inverses of square matrices as outer inverses with prescribed null space and range. In addition, we characterize the core EP inverse as the solution of a new system of matrix equations.
\end{abstract}

AMS Classification: 15A09

Keywords: Core inverse, core EP inverse, DMP inverse, CMP inverse, $k$-commutative equalities.

\section{Introduction}

Let $\mathbb{C}^{m \times n}$ be the set of $m \times n$ complex matrices. For $A \in \mathbb{C}^{m \times n}$, the symbols $A^{*}, A^{-1}, \operatorname{rk}(A), \mathcal{N}(A)$, and $\mathcal{R}(A)$ will denote the conjugate transpose, the inverse $(m=n)$, the rank, the kernel, and the range space of $A$, respectively. Moreover, $I_{n}$ will refer to the $n \times n$ identity matrix.

Let $A \in \mathbb{C}^{m \times n}$. We recall that the unique matrix $X \in \mathbb{C}^{n \times m}$ satisfying

$$
A X A=A, \quad X A X=X, \quad(A X)^{*}=A X, \quad \text { and } \quad(X A)^{*}=X A
$$

*Universidad Nacional de Río Cuarto, CONICET, FCEFQyN, RN 36 Km 601, Río Cuarto, 5800, Córdoba. Argentina. E-mail: deferreyra@exa.unrc.edu.ar, flevis@exa.unrc.edu.ar. Partially supported by a CONICET's Posdoctoral Research Fellowship, UNRC (grant PPI 18/C472), UNLPam, Facultad de Ingeniería (grant Resol. No 155/14) and CONICET (grant PIP 112-201501-00433CO).

${ }^{\dagger}$ Instituto Universitario de Matemática Multidisciplinar, Universitat Politècnica de València, 46022, Valencia, Spain. E-mail: njthome@mat.upv.es. Partially supported by Ministerio de Economía, Industria y Competitividad of Spain (Grant DGI MTM2013-43678-P and Grant Red de Excelencia MTM2017-90682-REDT). 
is called the Moore-Penrose inverse of $A$ and is denoted by $A^{\dagger}$. A matrix $X \in \mathbb{C}^{n \times m}$ that satisfies the equality $A X A=A$ is called an inner inverse or $\{1\}$-inverse of $A$, and a matrix $X \in \mathbb{C}^{n \times m}$ that satisfies the equality $X A X=X$ is called an outer inverse or $\{2\}$-inverse of $A$.

For a given complex square matrix $A$, the index of $A$, denoted by $\operatorname{Ind}(A)$, is the smallest nonnegative integer $k$ such that $\mathcal{R}\left(A^{k}\right)=\mathcal{R}\left(A^{k+1}\right)$. We observe that the index of a nonsingular matrix $A$ is 0 , and by convention, the index of the null matrix is 1 . We also recall that the Drazin inverse of $A \in \mathbb{C}^{n \times n}$ is the unique matrix $X \in \mathbb{C}^{n \times n}$ such that $X A X=X, A X=X A$, and $A^{k+1} X=A^{k}$, where $k=\operatorname{Ind}(A)$, and is denoted by $A^{d}$. If $A \in \mathbb{C}^{n \times n}$ satisfies $\operatorname{Ind}(A) \leq 1$, then the Drazin inverse of $A$ is called the group inverse of $A$ and is denoted by $A^{\#}$.

The core inverse was introduced by Baksalary and Trenkler in [2]. For a given matrix $A \in \mathbb{C}^{n \times n}$, its core inverse is the unique matrix $X \in \mathbb{C}^{n \times n}$ defined by the conditions $A X=P_{A}$ and $\mathcal{R}(X) \subseteq \mathcal{R}(A)$, where $P_{A}$ is the orthogonal projector onto the range of $A$, i.e., $P_{A}=A A^{\dagger}$. In case that such a matrix $X$ exists, it is denoted by $A^{\oplus}$. Moreover, it was proved that $A$ is core invertible if and only if $\operatorname{Ind}(A) \leq 1$.

Two generalizations of the core inverse have been recently introduced for complex square matrices. Recall, for a given matrix $A \in \mathbb{C}^{n \times n}$ of index $k$, the unique matrix $X \in \mathbb{C}^{n \times n}$ such that

$$
X A X=X \quad \text { and } \quad \mathcal{R}(X)=\mathcal{R}\left(X^{*}\right)=\mathcal{R}\left(A^{k}\right),
$$

is called the core EP inverse of $A$ and is denoted by $A^{\oplus}[8]$. The authors proved that the core EP of a matrix $A \in \mathbb{C}^{n \times n}$ is the unique solution of

$$
X A^{k+1}=A^{k}, \quad X A X=X, \quad(A X)^{*}=A X, \quad \text { and } \quad \mathcal{R}(X) \subseteq \mathcal{R}\left(A^{k}\right),
$$

[8, Lemma 3.3]. Notice that equations in (2) are equivalent to a new set of equations containing the same first three and changing the inclusion $\mathcal{R}(X) \subseteq \mathcal{R}\left(A^{k}\right)$ with the equality $\mathcal{R}(X)=\mathcal{R}\left(A^{k}\right)$. Secondly, the concept of DMP inverse of $A$ was introduced in [5]. In this case, the unique matrix $X \in \mathbb{C}^{n \times n}$ satisfying

$$
X A X=X, \quad X A=A^{d} A, \quad \text { and } \quad A^{k} X=A^{k} A^{\dagger},
$$

is called the DMP inverse of $A$ and is denoted by $A^{d, \dagger}$. Moreover, it was proved that $A^{d, \dagger}=A^{d} A A^{\dagger}$. The authors introduced also another outer inverse associated to a square matrix, namely $A^{\dagger, d}=A^{\dagger} A A^{d}$ called dual DMP inverse of $A$.

Recently, a new generalized inverse was given in [7]. In this case, the matrix

$$
A^{c, \dagger}:=Q_{A} A^{d} P_{A}, \quad \text { where } \quad Q_{A}=A^{\dagger} A,
$$

is called the CMP inverse of $A$. 
We recall that a matrix $A \in \mathbb{C}^{n \times n}$ is EP if $A A^{\dagger}=A^{\dagger} A$. In [6] the authors introduced $k$-EP matrices mimicking the idea of EP matrices, in this case for $k>1$. A matrix $A \in \mathbb{C}^{n \times n}$ of index $k$ is called $k$-EP matrix if $A^{k} A^{\dagger}=A^{\dagger} A^{k}$, that is,

$$
\mathbb{C}_{n}^{k, \dagger}=\left\{A \in \mathbb{C}^{n \times n}: A^{k} A^{\dagger}=A^{\dagger} A^{k}\right\}
$$

Let $A \in \mathbb{C}^{n \times n}$. Throughout all the paper we will assume that $\operatorname{Ind}(A)=k \geq 1$. In this paper we are going to study the class

$$
\mathbb{C}_{n}^{k}=\left\{X \in \mathbb{C}^{n \times n}: A^{k} X=X A^{k}, X A X=X\right\}
$$

and the stress will be put on a sort of inverse problems by considering the following classes of matrices:

$$
\begin{aligned}
& \mathbb{C}_{n}^{k, \oplus}=\left\{A \in \mathbb{C}^{n \times n}: A^{k} A^{\oplus}=A^{\oplus} A^{k}\right\}, \\
& \mathbb{C}_{n}^{k, d \dagger}=\left\{A \in \mathbb{C}^{n \times n}: A^{k} A^{d, \dagger}=A^{d, \dagger} A^{k}\right\}, \\
& \mathbb{C}_{n}^{k, \dagger d}=\left\{A \in \mathbb{C}^{n \times n}: A^{k} A^{\dagger, d}=A^{\dagger, d} A^{k}\right\}, \\
& \mathbb{C}_{n}^{k, c \dagger}=\left\{A \in \mathbb{C}^{n \times n}: A^{k} A^{c, \dagger}=A^{c, \dagger} A^{k}\right\},
\end{aligned}
$$

which lead us to new generalizations for EP matrices. The matrices in these last classes will be called $k$-core EP, $k$-DMP, dual $k$-DMP and $k$-CMP matrices, respectively.

This paper is organized as follows. In Section 2, a necessary and sufficient condition characterizing the class matrices $A$ such that $A^{k} X=X A^{k}$, for $X$ being an outer inverse of $A$, is given. Section 3 presents new representations of core EP inverse, DMP and dual DMP inverses, and CMP inverse of a square matrix as an outer inverse with prescribed range and null space. For further investigations, we also derive representations for the Drazin inverse, the Moore-Penrose inverse, the DMP inverse, the dual DMP inverse, and the CMP inverse in terms of the core EP decomposition. In addition, we state new characterizations of $k$-EP matrices by using the recent core EP decomposition given by Wang in [10]. Similarly, we give characterizations of $k$-core EP matrices, $k$-DMP matrices, dual $k$-DMP matrices, and $k$-CMP matrices. As a consequence, we derive that the class $k$-EP is (properly) included in both $k$-DMP and dual $k$-DMP classes. Finally, Section 4 provides characterizations of core EP matrices by means of a new set of matrix equations. This new set reduces from four to three the number of equations given by Prasad and Mohana in [8] showing that the first equation in (2) is redundant. 


\section{$2 \quad$ The general class $\mathbb{C}_{n}^{k}$}

Related to power of matrices commuting with generalized inverses, for a given $A \in \mathbb{C}^{n \times n}$ of index at most 1 , the problem of characterizing all matrices $X \in \mathbb{C}^{n \times n}$ such that

$$
A^{k} X^{k} A^{k}=A^{k} \quad \text { and } \quad X^{k} A^{k} X^{k}=X^{k}, \text { for all } k \in \mathbb{N}
$$

was studied in Rao and Mitra's book [13, p. 77]. Using the canonical form for index 1 matrices,

$$
A=L\left[\begin{array}{ll}
C & 0 \\
0 & 0
\end{array}\right] L^{-1},
$$

for $C$ and $L$ being nonsingular, the most general form of $X$ 's satisfying both conditions in (5) is given by

$$
X=L\left[\begin{array}{cc}
C^{-1} & J \\
F & F C J
\end{array}\right] L^{-1}
$$

provided that $F$ and $J$ fulfill $J F=0$. Notice that both conditions in (5) are true for $\chi$-inverses and $\rho$-inverses [13, pp. 73 and 77].

Let $A \in \mathbb{C}^{n \times n}$ and assume that $\operatorname{Ind}(A)=k \geq 1$. According to Theorem 2.2 in [10], every matrix $A \in \mathbb{C}^{n \times n}$ with $\operatorname{Ind}(A)=k$ can be represented in the form

$$
A=A_{1}+A_{2}, \quad A_{1}:=U\left[\begin{array}{cc}
T & S \\
0 & 0
\end{array}\right] U^{*}, \quad A_{2}:=U\left[\begin{array}{cc}
0 & 0 \\
0 & N
\end{array}\right] U^{*},
$$

where $T$ is nonsingular with $t:=\operatorname{rk}(T)=\operatorname{rk}\left(A^{k}\right), N$ is nilpotent of index $k$, and $U$ is unitary. The representation of $A$ given in (6) satisfies $\operatorname{Ind}\left(A_{1}\right) \leq 1, A_{2}^{k}=0$, and $A_{1}^{*} A_{2}=A_{2} A_{1}=0$ [10, Theorem 2.1]. Moreover, it is unique [10, Theorem 2.4] and is called the core EP decomposition of $A$. The notation

$$
\widetilde{T}=\sum_{j=0}^{k-1} T^{j} S N^{k-1-j}
$$

will be used in the forthcoming results.

The symbol $A\{2\}$ stands for the set of all $\{2\}$-inverses of $A$ and $A\{5\}$ denotes the set of all matrices commuting with $A$. Next result completely describes the set $\mathbb{C}_{n}^{k}$.

Theorem 2.1. Let $A \in \mathbb{C}^{n \times n}$ written as in (6) and $k \geq 1$ be the index of $A$. Then $X \in \mathbb{C}_{n}^{k}$ if and only if

$$
X=U\left[\begin{array}{cc}
X_{1} & T^{-k}\left(X_{1} \widetilde{T}-\widetilde{T} X_{4}\right) \\
0 & X_{4}
\end{array}\right] U^{*},
$$

where $X_{1} \in T\{2\} \cap T^{k}\{5\}$ and $X_{4} \in N\{2\}$. 
Proof. From

$$
A=U\left[\begin{array}{ll}
T & S \\
0 & N
\end{array}\right] U^{*},
$$

and using that $A$ has index $k$ and (7), it is clear that

$$
A^{k}=U\left[\begin{array}{cc}
T^{k} & \widetilde{T} \\
0 & 0
\end{array}\right] U^{*}
$$

We partition

$$
X=U\left[\begin{array}{ll}
X_{1} & X_{2} \\
X_{3} & X_{4}
\end{array}\right] U^{*}
$$

accordingly to the sizes of the blocks of $A$. From $A^{k} X=X A^{k}$ we obtain

$$
\left[\begin{array}{cc}
T^{k} X_{1}+\widetilde{T} X_{3} & T^{k} X_{2}+\widetilde{T} X_{4} \\
0 & 0
\end{array}\right]=\left[\begin{array}{cc}
X_{1} T^{k} & X_{1} \widetilde{T} \\
X_{3} T^{k} & X_{3} \widetilde{T}
\end{array}\right]
$$

from where $T^{k} X_{1}=X_{1} T^{k}, X_{3}=0$, and $X_{2}=T^{-k}\left(X_{1} \widetilde{T}-\widetilde{T} X_{4}\right)$.

Now, using that $X A X=X$ we arrive at $X_{1} T X_{1}=X_{1}, X_{4} N X_{4}=X_{4}$.

We observe that the equation

$$
T^{-k}\left(X_{1} \widetilde{T}-\widetilde{T} X_{4}\right)=X_{1} T^{-k} T\left(X_{1} \widetilde{T}-\widetilde{T} X_{4}\right)+X_{1} S X_{4}+T^{-k}\left(X_{1} \widetilde{T}-\widetilde{T} X_{4}\right) N X_{4}
$$

is always true due to $T^{-k} X_{1}=X_{1} T^{-k}$ and because if we focus on the powers of the expression of $\widetilde{T}$, it is easy to show that $\widetilde{T} N-T \widetilde{T}=-T^{k} S$. The converse is evident.

The rest of the paper is devoted to investigate all the square matrices $A$ of index $k \geq 1$ satisfying

$$
A^{k} X=X A^{k} \quad \text { for } \quad X \in\left\{A^{\dagger}, A^{\oplus}, A^{d, \dagger}, A^{\dagger, d}, A^{c, \dagger}\right\} .
$$

In [15], Wang and Chen introduced the weak group inverse of a matrix $A \in \mathbb{C}^{n \times n}$ of index $k$ as the unique matrix $X \in \mathbb{C}^{n \times n}$ satifying $A X^{2}=X$ and $A X=A^{\oplus} A$ and it was denoted by $X=A^{\circledR}$. The authors shown that if $A$ is written in the core EP decomposition (6) then

$$
A^{\circledR}=U\left[\begin{array}{cc}
T^{-1} & T^{-2} S \\
0 & 0
\end{array}\right] U^{*} .
$$

In spite of the weak group inverse $A^{\circledR}$ is not an outer inverse of $A$, we can state the following result.

Lemma 2.2. Let $A \in \mathbb{C}^{n \times n}$ with $\operatorname{Ind}(A)=k$ written as in (6). Then $A^{k} A^{\bowtie}=A^{\bowtie} A^{k}$ if and only if $\sum_{j=0}^{k-2} T^{j} S N^{k-1-j}=0$.

Proof. It is a simple computation that follows from (6), (8), and using the nonsingularity of $T$. 


\section{Representations of core EP, DMP and dual DMP, and CMP inverses}

Let $A \in \mathbb{C}^{n \times n}$ a matrix of rank $r$. Let $T$ be a subspace of $\mathbb{C}^{n}$ of dimension $s \leq r$, and let $S$ be a subspace of $\mathbb{C}^{n}$ of dimension $n-s$. It is well known that $A$ has a $\{2\}$-inverse $X$ such that $\mathcal{R}(X)=T$ and $\mathcal{N}(X)=S$ if and only if $A T \oplus S=\mathbb{C}^{n}$, in which case $X$ is unique and is denoted by $A_{T, S}^{(2)}[1$, Theorem 14, p. 72]. Moreover, if $B \in \mathbb{C}^{n \times n}$ satisfies $\mathcal{R}(B)=T, \mathcal{N}(B)=S$ and $A$ has an inverse $A_{T, S}^{(2)}$ then $\operatorname{Ind}(A B) \leq 1$ and $\operatorname{Ind}(B A) \leq 1$. Furthermore, we have $A_{T, S}^{(2)}=(B A)^{\#} B$ and $A_{T, S}^{(2)}=B(A B)^{\#}$ [16, Theorem 2.1].

Recall that the Moore-Penrose inverse, the Drazin inverse, and the group inverse are $\{2\}$-inverses of $A$ with prescribed range and null space satisfying

$$
A^{\dagger}=A_{\mathcal{R}\left(A^{*}\right), \mathcal{N}\left(A^{*}\right)}^{(2)}, \quad A^{\#}=A_{\mathcal{R}(A), \mathcal{N}(A)}^{(2)}, \quad \text { and } \quad A^{d}=A_{\mathcal{R}\left(A^{k}\right), \mathcal{N}\left(A^{k}\right)}^{(2)}
$$

Also, it was proved in [11] that $A^{\boxplus}=A_{\mathcal{R}(A), \mathcal{N}\left(A^{*}\right)}^{(2)}$ holds. For similar results extended to weighted inverses we refer the reader to $[12,14]$.

Remark 3.1. We observe that if $X$ is an outer inverse of $A$, then $\mathcal{N}(A X) \subseteq \mathcal{N}(X A X)=\mathcal{N}(X) \subseteq$ $\mathcal{N}(A X)$ and $\mathcal{R}(X A) \subseteq \mathcal{R}(X)=\mathcal{R}(X A X) \subseteq \mathcal{R}(X A)$, i.e., $\mathcal{N}(X)=\mathcal{N}(A X)$ and $\mathcal{R}(X)=\mathcal{R}(X A)$. That is, the inverse $A_{\mathcal{R}(X), \mathcal{N}(X)}^{(2)}$ exists. Thus, $\mathbb{C}^{n}=A \mathcal{R}(X) \oplus \mathcal{N}(X)$.

In the following result we give new representations of core EP inverses, DMP inverses and CMP inverses. From now on, the symbol $A_{c}$ stands for the product $A A^{d} A$, which represents the core part of the core-nilpotent decomposition of the matrix $A$, that is $A^{(c)}:=A A^{d} A$.

Theorem 3.2. Let $A \in \mathbb{C}^{n \times n}$ with $\operatorname{Ind}(A)=k$. Then

(a) $A^{\oplus}=A_{\mathcal{R}\left(A^{k}\right), \mathcal{N}\left(\left(A^{k}\right)^{*}\right)}^{(2)}$;

(b) $A^{d, \dagger}=A_{\mathcal{R}\left(A^{k}\right), \mathcal{N}\left(A^{k} A^{\dagger}\right)}^{(2)}$;

(c) $A^{c, \dagger}=A_{\mathcal{R}\left(A^{\dagger} A^{k}\right), \mathcal{N}\left(A^{k} A^{\dagger}\right)}^{(2)}$;

(d) $A^{\dagger, d}=A_{\mathcal{R}\left(A^{\dagger} A^{k}\right), \mathcal{N}\left(A^{k}\right)}^{(2)}$.

Proof. We first notice that, by definition, each of $A^{\oplus}, A^{d, \dagger}, A^{c, \dagger}$, and $A^{\dagger, d}$ are outer inverses of $A$.

(a) We recall that $\mathcal{R}\left(A^{\oplus}\right)=\mathcal{R}\left(A^{k}\right)$. From [4, Theorem 3.7] we know that $\mathcal{N}\left(A A^{\oplus}\right)=\mathcal{N}\left(\left(A^{k}\right)^{*}\right)$. From Remark 3.1 we have $\mathcal{N}\left(A^{\oplus}\right)=\mathcal{N}\left(\left(A^{k}\right)^{*}\right)$. Hence, by [1, Theorem 14, pp. 72] we obtain $(a)$.

(b) From [5, Theorem 2.12] we know that $\mathcal{R}\left(A^{d, \dagger} A\right)=\mathcal{R}\left(A^{k}\right)$ and $\mathcal{N}\left(A A^{d, \dagger}\right)=\mathcal{N}\left(A^{d} A A^{\dagger}\right)$. Since 
$\mathcal{N}\left(A^{d} A\right)=\mathcal{N}\left(A^{k}\right)$ it is clear that $\mathcal{N}\left(A^{d} A A^{\dagger}\right)=\mathcal{N}\left(A^{k} A^{\dagger}\right)$. Now, From Remark 3.1 we have $\mathcal{R}\left(A^{d, \dagger}\right)=$ $\mathcal{R}\left(A^{k}\right)$ and $\mathcal{N}\left(A^{d, \dagger}\right)=\mathcal{N}\left(A^{k} A^{\dagger}\right)$. In consequence, Remark 3.1 and [1, Theorem 14, p. 72] complete the proof.

(c) From Remark 3.1 and the definition of $A^{c, \dagger}$ we have $\mathcal{N}\left(A^{c, \dagger}\right)=\mathcal{N}\left(A A^{c, \dagger}\right)=\mathcal{N}\left(A^{(c)} A^{c, \dagger}\right)$. Since $A^{c, \dagger}=A^{\dagger} A^{(c)} A^{\dagger}=A^{\dagger} A A^{d} A A^{\dagger}$ then

$$
\mathcal{N}\left(A^{c, \dagger}\right)=\mathcal{N}\left(A A^{d} A A^{\dagger}\right)=\mathcal{N}\left(A A^{d, \dagger}\right)=\mathcal{N}\left(A^{d, \dagger}\right)=\mathcal{N}\left(A^{k} A^{\dagger}\right)
$$

where the last equality follows from (b). On the other hand, by definition of $A^{c, \dagger}$ and Remark 3.1 we also have $\mathcal{R}\left(A^{c, \dagger}\right)=\mathcal{R}\left(A^{c, \dagger} A\right)=\mathcal{R}\left(A^{\dagger} A^{(c)}\right)=\mathcal{R}\left(A^{\dagger} A A^{d} A\right)=\mathcal{R}\left(A^{\dagger, d} A\right)=\mathcal{R}\left(A^{\dagger, d}\right)$, where the last equality uses the fact that $A^{\dagger, d}$ is a $\{2\}$-inverse of $A$. Now, as $\mathcal{R}\left(A^{\dagger, d}\right)=A^{\dagger} \mathcal{R}\left(A A^{d}\right)$ and $\mathcal{R}\left(A A^{d}\right)=\mathcal{R}\left(A^{k}\right)$, we obtain $\mathcal{R}\left(A^{c, \dagger}\right)=\mathcal{R}\left(A^{\dagger} A^{k}\right)$. Finally, (c) follows from Remark 3.1 and [1, Theorem 14, pp. 72].

(d) As in the proof of (c) we have $\mathcal{R}\left(A^{\dagger, d}\right)=\mathcal{R}\left(A^{\dagger} A^{k}\right)$. On the other hand, from Remark 3.1, $\mathcal{N}\left(A^{\dagger, d}\right)=\mathcal{N}\left(A A^{\dagger, d}\right)=\mathcal{N}\left(A A^{\dagger} A A^{d}\right)=\mathcal{N}\left(A A^{d}\right)=\mathcal{N}\left(A^{k}\right)$. Therefore, we arrive at (d) by using Remark 3.1 and [1, Theorem 14, p. 72].

Corollary 3.3. Let $A \in \mathbb{C}^{n \times n}$ with $\operatorname{Ind}(A)=k$. Then the following statements hold:

(a) $A^{c, \dagger}=A^{\dagger, d}$ if and only if $\mathcal{N}\left(A^{k} A^{\dagger}\right)=\mathcal{N}\left(A^{k}\right)$;

(b) $A^{c, \dagger}=A^{d, \dagger}$ if and only if $\mathcal{R}\left(A^{\dagger} A^{k}\right)=\mathcal{R}\left(A^{k}\right)$;

(c) $A^{\oplus}=A^{d, \dagger}$ if and only if $\mathcal{N}\left(A^{k} A^{\dagger}\right)=\mathcal{N}\left(\left(A^{k}\right)^{*}\right)$.

(d) $A^{\oplus}=A^{\dagger, d}$ if and only if $A^{k}$ is EP and $\mathcal{R}\left(A^{k}\right)$ is $A^{\dagger}$-invariant.

Theorem 3.4. Let $A \in \mathbb{C}^{n \times n}$ with $\operatorname{Ind}(A)=k$. Then

$$
A^{\oplus}=\left(P_{A^{k}} A\right)^{\#} P_{A^{k}}=P_{A^{k}}\left(A P_{A^{k}}\right)^{\#} .
$$

Proof. Since $P_{A^{k}}=A^{k}\left(A^{k}\right)^{\dagger}$ is an orthogonal projector on $\mathcal{R}\left(A^{k}\right)$, we have

$$
\mathcal{R}\left(A^{\oplus}\right)=\mathcal{R}\left(A^{k}\right)=\mathcal{R}\left(P_{A^{k}}\right),
$$

From [10, Corollary 3.3] we obtain

$$
\mathcal{N}\left(P_{A^{k}}\right)=\mathcal{N}\left(A A^{\oplus}\right) \subseteq \mathcal{N}\left(A^{\oplus} A A^{\oplus}\right)=\mathcal{N}\left(A^{\oplus}\right) \subseteq \mathcal{N}\left(A A^{\oplus}\right)=\mathcal{N}\left(P_{A^{k}}\right) .
$$

Consequently, (10) follows from (11), (12), Theorem 3.2, and [16, Theorem 2.1] with $B=P_{A^{k}}$.

Remark 3.5. When $\operatorname{Ind}(A) \leq 1$, from the representation given in (10), it is easy to verify that $A^{\boxplus}=A^{\#} P_{A}[2$, Theorem 1]. 
Remark 3.6. It is easily verified that $A^{\oplus}=\left(A^{\oplus} A\right)^{\#} A^{\oplus}=A^{\oplus}\left(A A^{\oplus}\right)^{\#}$ by setting $B=A^{\oplus}$ in the definition of $A_{T, S}^{(2)}$ and by using Theorem $3.2(a)$. Observe the similarity of formulae for $A^{\oplus}$ and $A^{\dagger}$ by noting that $A^{\dagger}=\left(A^{*} A\right)^{\dagger} A^{*}=A^{*}\left(A A^{*}\right)^{\dagger}$.

Wang [10, Theorem 13] also gave a representation for the core EP inverse. More precisely, for a matrix $A$ represented as in (6), its core EP inverse is given by

$$
A^{\oplus}=U\left[\begin{array}{cc}
T^{-1} & 0 \\
0 & 0
\end{array}\right] U^{*} \text {. }
$$

Now, we give a new representation for Drazin matrices by using the core EP decomposition.

Theorem 3.7. Let $A \in \mathbb{C}^{n \times n}$ be a matrix of index $k$ written as in (6). Then

$$
A^{d}=U\left[\begin{array}{cc}
T^{-1} & \left(T^{k+1}\right)^{-1} \widetilde{T} \\
0 & 0
\end{array}\right] U^{*}
$$

Proof. If we write $A$ as in (6) and recall (7) then

$$
A^{k}=U\left[\begin{array}{cc}
T^{k} & \widetilde{T} \\
0 & 0
\end{array}\right] U^{*}
$$

As $A^{k+1}=A^{k} A=A A^{k}$, a straightforward computation shows that

$$
A^{k+1}=U\left[\begin{array}{cc}
T^{k+1} & T^{k} S+\widetilde{T} N \\
0 & 0
\end{array}\right] U^{*}=U\left[\begin{array}{cc}
T^{k+1} & T \widetilde{T} \\
0 & 0
\end{array}\right] U^{*} .
$$

Then,

$$
T^{k} S+\widetilde{T} N=T \widetilde{T} \quad \text { or equivalently }\left(T^{k}\right)^{-1} \widetilde{T}=T^{-1} S+\left(T^{k+1}\right)^{-1} \widetilde{T} N
$$

Let

$$
X=U\left[\begin{array}{cc}
T^{-1} & \left(T^{k+1}\right)^{-1} \widetilde{T} \\
0 & 0
\end{array}\right] U^{*}
$$

Now, we shall prove that the matrix $X$ satisfies the system $X A X=X, A X=X A$, and $A^{k+1} X=A^{k}$. In fact,

$$
A^{k+1} X=U\left[\begin{array}{cc}
T^{k+1} & T \widetilde{T} \\
0 & 0
\end{array}\right]\left[\begin{array}{cc}
T^{-1} & \left(T^{k+1}\right)^{-1} \widetilde{T} \\
0 & 0
\end{array}\right] U^{*}=U\left[\begin{array}{cc}
T^{k} & \widetilde{T} \\
0 & 0
\end{array}\right] U^{*}=A^{k} .
$$

From (16) we get

$$
\begin{aligned}
A X & =U\left[\begin{array}{ll}
T & S \\
0 & N
\end{array}\right]\left[\begin{array}{cc}
T^{-1} & \left(T^{k+1}\right)^{-1} \widetilde{T} \\
0 & 0
\end{array}\right] U^{*}=U\left[\begin{array}{cc}
I_{t} & \left(T^{k}\right)^{-1} \widetilde{T} \\
0 & 0
\end{array}\right] U^{*} \\
& =U\left[\begin{array}{cc}
I_{t} & T^{-1} S+\left(T^{k+1}\right)^{-1} \widetilde{T} N \\
0 & 0
\end{array}\right] U^{*}=U\left[\begin{array}{cc}
T^{-1} & \left(T^{k+1}\right)^{-1} \widetilde{T} \\
0 & 0
\end{array}\right]\left[\begin{array}{cc}
T & S \\
0 & N
\end{array}\right] U^{*}=X A .
\end{aligned}
$$


Finally,

$$
X A X=U\left[\begin{array}{cc}
T^{-1} & \left(T^{k+1}\right)^{-1} \widetilde{T} \\
0 & 0
\end{array}\right]\left[\begin{array}{cc}
I_{t} & \left(T^{k}\right)^{-1} \widetilde{T} \\
0 & 0
\end{array}\right] U^{*}=U\left[\begin{array}{cc}
T^{-1} & \left(T^{k+1}\right)^{-1} \widetilde{T} \\
0 & 0
\end{array}\right] U^{*}=X
$$

Since the Drazin inverse is unique, we conclude that $X=A^{d}$ holds.

Corollary 3.8. Let $A \in \mathbb{C}^{n \times n}$ with $\operatorname{Ind}(A)=k$. Then

$$
A^{\oplus}=A^{d} P_{A^{k}} .
$$

Proof. Let $A \in \mathbb{C}^{n \times n}$ be written as in (6). By [4, Lemma 2.5] we obtain

$$
P_{A^{k}}=A^{k}\left(A^{k}\right)^{\dagger}=U\left[\begin{array}{cc}
I_{t} & 0 \\
0 & 0
\end{array}\right] U^{*}
$$

On the other hand, by [10, Theorem 3.2] we have that

$$
A^{\oplus}=U\left[\begin{array}{cc}
T^{-1} & 0 \\
0 & 0
\end{array}\right] U^{*} \text {. }
$$

Hence, from Theorem 3.7 and (18) we obtain (17).

In [3], the authors found the Moore-Penrose inverse of a linear operator for which its matrix block representation is block (upper) triangular with some diagonal block being nonsingular. The following result provides a representation for the Moore Penrose inverse by using the core EP decomposition for a general matrix. The importance of this result lies in the fact that it is valid with no extra restrictions to be assumed which highlight the power of the core EP decomposition.

Theorem 3.9. Let $A \in \mathbb{C}^{n \times n}$ be a matrix of index $k$ written as in (6). Then

$$
A^{\dagger}=U\left[\begin{array}{cc}
T^{*} \Delta & -T^{*} \Delta S N^{\dagger} \\
\left(I_{n-t}-N^{\dagger} N\right) S^{*} \Delta & N^{\dagger}-\left(I_{n-t}-N^{\dagger} N\right) S^{*} \Delta S N^{\dagger}
\end{array}\right] U^{*}
$$

where $\Delta=\left(T T^{*}+S\left(I_{n-t}-N^{\dagger} N\right) S^{*}\right)^{-1}$.

Proof. The proof follows immediately from [1, Ex. 25, p. 49] and [3, Lemma 6].

Define the matrix

$$
\left[\begin{array}{cc}
R & V \\
W & Z
\end{array}\right]:=\left[\begin{array}{cc}
T^{*} \Delta & -T^{*} \Delta S N^{\dagger} \\
\left(I_{n-t}-N^{\dagger} N\right) S^{*} \Delta & N^{\dagger}-\left(I_{n-t}-N^{\dagger} N\right) S^{*} \Delta S N^{\dagger}
\end{array}\right],
$$

in order to consider the central block obtained in (19).

Next, we establish a new geometrical characterization for $k$-EP matrices by using the core EP decomposition. We recall that $A$ is $k$-EP if and only if $A \in \mathbb{C}_{n}^{k, \dagger}$. 
Theorem 3.10. Let $A \in \mathbb{C}^{n \times n}$ be a matrix of index $k$ written as in (6). Then $A \in \mathbb{C}_{n}^{k, \dagger}$ if and only if the following conditions simultaneously hold:

(i) $\mathcal{N}(N) \subseteq \mathcal{N}(S)$ (or equivalently $S\left(I_{n-t}-N^{\dagger} N\right)=0$ ),

(ii) $\mathcal{N}\left(N^{*}\right) \subseteq \mathcal{N}(\widetilde{T})$ (or equivalently $\widetilde{T}\left(I_{n-t}-N N^{\dagger}\right)=0$ ).

Proof. Let $A \in \mathbb{C}^{n \times m}$ be written as in (6) and consider the expression for $A^{\dagger}$ given in (19). Assuming that $A$ is $k$-EP, from (15) and (20) we have

$$
\left[\begin{array}{cc}
R T^{k} & R \widetilde{T} \\
W T^{k} & W \widetilde{T}
\end{array}\right]=\left[\begin{array}{cc}
T^{k} R+\widetilde{T} W & T^{k} V+\widetilde{T} Z \\
0 & 0
\end{array}\right] .
$$

Since $T$ is nonsigular, $W=0$. Also, since $\Delta$ is nonsingular, we have $\left(I_{n-t}-N^{\dagger} N\right) S^{*}=0$ or equivalently $S\left(I_{n-t}-N^{\dagger} N\right)=0$ holds. Observe that this last equality holds if and only if $\mathcal{N}(N)=\mathcal{N}\left(N^{\dagger} N\right)=$ $\mathcal{R}\left(I_{n-t}-N^{\dagger} N\right) \subseteq \mathcal{N}(S)$. So, from Theorem 3.9, we obtain $\Delta=\left(T^{*}\right)^{-1} T^{-1}$ and consequently $R=T^{-1}$, $V=-T^{-1} S N^{\dagger}, Z=N^{\dagger}$, and $R \widetilde{T}=T^{k} V+\widetilde{T} Z$. Thus, $T^{-1} \widetilde{T}=-T^{k-1} S N^{\dagger}+\widetilde{T} N^{\dagger}$ or equivalently $\widetilde{T}=-T^{k} S N^{\dagger}+T \widetilde{T} N^{\dagger}$. According to (16) we have $\widetilde{T}=-T^{k} S N^{\dagger}+\left(\widetilde{T} N+T^{k} S\right) N^{\dagger}=\widetilde{T} N N^{\dagger}$, which implies $\widetilde{T}\left(I_{n-t}-N N^{\dagger}\right)=0$. Equivalently, $\mathcal{N}\left(N^{*}\right)=\mathcal{N}\left(N^{\dagger}\right)=\mathcal{N}\left(N N^{\dagger}\right)=\mathcal{R}\left(I_{n-t}-N N^{\dagger}\right) \subseteq \mathcal{N}(\widetilde{T})$. Conversely, we suppose that $S\left(I_{n-t}-N^{\dagger} N\right)=0$ and $\widetilde{T}\left(I_{n-t}-N N^{\dagger}\right)=0$ hold. Since $\left(I_{n-t}-N^{\dagger} N\right) S^{*}=$ 0 , from Theorem 3.9 we deduce that $\Delta=\left(T^{*}\right)^{-1} T^{-1}$ and so

$$
A^{\dagger}=U\left[\begin{array}{cc}
T^{-1} & -T^{-1} S N^{\dagger} \\
0 & N^{\dagger}
\end{array}\right] U^{*} .
$$

From (16), it follows that $-T^{k-1} S N^{\dagger}+\widetilde{T} N^{\dagger}=-T^{-1} T^{k} S N^{\dagger}+T^{-1} T \widetilde{T} N^{\dagger}=T^{-1}\left(T \widetilde{T}-T^{k} S\right) N^{\dagger}=$ $T^{-1} \widetilde{T} N N^{\dagger}=T^{-1} \widetilde{T}$. Therefore,

$$
\begin{aligned}
A^{k} A^{\dagger} & =U\left[\begin{array}{cc}
T^{k} & \widetilde{T} \\
0 & 0
\end{array}\right]\left[\begin{array}{cc}
T^{-1} & -T^{-1} S N^{\dagger} \\
0 & N^{\dagger}
\end{array}\right] U^{*}=U\left[\begin{array}{cc}
T^{k-1} & -T^{k-1} S N^{\dagger}+\widetilde{T} N^{\dagger} \\
0 & 0
\end{array}\right] U^{*}, \\
& =U\left[\begin{array}{cc}
T^{k-1} & T^{-1} \widetilde{T} \\
0 & 0
\end{array}\right] U^{*}=U\left[\begin{array}{cc}
T^{-1} & -T^{-1} S N^{\dagger} \\
0 & N^{\dagger}
\end{array}\right]\left[\begin{array}{cc}
T^{k} & \widetilde{T} \\
0 & 0
\end{array}\right] U^{*}=A^{\dagger} A^{k} .
\end{aligned}
$$

i.e., $A$ is a $k$-EP matrix.

In order to obtain similar results for the sets $\mathbb{C}_{n}^{k, d \dagger}$ and $\mathbb{C}_{n}^{k, \dagger d}$ we need representations for $A^{d, \dagger}$ and $A^{\dagger, d}$ given by means of core EP factorization of $A$.

Theorem 3.11. Let $A \in \mathbb{C}^{n \times n}$ be a matrix of index $k$ written as in (6). Then

$$
A^{d, \dagger}=U\left[\begin{array}{cc}
T^{-1} & \left(T^{k+1}\right)^{-1} \widetilde{T} N N^{\dagger} \\
0 & 0
\end{array}\right] U^{*}
$$


and

$$
A^{\dagger, d}=U\left[\begin{array}{cc}
T^{*} \Delta & T^{*} \Delta T^{-k} \widetilde{T} \\
\left(I_{n-t}-N^{\dagger} N\right) S^{*} \Delta & \left(I_{n-t}-N^{\dagger} N\right) S^{*} \Delta T^{-k} \widetilde{T}
\end{array}\right] U^{*},
$$

where $\Delta$ is defined as in Theorem 3.9.

Proof. From (6), (19), and using the expression of $\Delta$, we have

$$
\begin{aligned}
A A^{\dagger} & =U\left[\begin{array}{cc}
{\left[T T^{*}+S\left(I_{n-t}-N^{\dagger} N\right) S^{*}\right] \Delta} & -T T^{*} \Delta S N^{\dagger}+S\left[N^{\dagger}-\left(I_{n-t}-N^{\dagger} N\right) S^{*} \Delta S N^{\dagger}\right] \\
N\left(I_{n-t}-N^{\dagger} N\right) S^{*} \Delta & N N^{\dagger}-N\left(I_{n-t}-N^{\dagger} N\right) S^{*} \Delta S N^{\dagger}
\end{array}\right] U^{*} \\
& =U\left[\begin{array}{cc}
{\left[T T^{*}+S\left(I_{n-t}-N^{\dagger} N\right) S^{*}\right] \Delta} & -\left[T T^{*}+S\left(I_{n-t}-N^{\dagger} N\right) S^{*}\right] \Delta S N^{\dagger}+S N^{\dagger} \\
0 & N N^{\dagger}
\end{array}\right] U^{*} \\
& =U\left[\begin{array}{cc}
I_{t} & 0 \\
0 & N N^{\dagger}
\end{array}\right] U^{*},
\end{aligned}
$$

and

$$
A^{\dagger} A=U\left[\begin{array}{cc}
T^{*} \Delta T & T^{*} \Delta S\left(I_{n-t}-N^{\dagger} N\right) \\
\left(I_{n-t}-N^{\dagger} N\right) S^{*} \Delta T & N^{\dagger} N+\left(I_{n-t}-N^{\dagger} N\right) S^{*} \Delta S\left(I_{n-t}-N^{\dagger} N\right)
\end{array}\right] U^{*} .
$$

Now, since $A^{d, \dagger}=A^{d} A A^{\dagger}$ and $A^{\dagger, d}=A^{\dagger} A A^{d}$, we have that (21) and (22) follow by using (14) and simple computations.

As a consequence, we derive a representation for CMP inverses.

Corollary 3.12. Let $A \in \mathbb{C}^{n \times n}$ be a matrix of index $k$ written as in (6). Then

$$
A^{c, \dagger}=U\left[\begin{array}{cc}
T^{*} \Delta & T^{*} \Delta\left(T^{k}\right)^{-1} \widetilde{T} N N^{\dagger} \\
\left(I_{n-t}-N^{\dagger} N\right) S^{*} \Delta & \left(I_{n-t}-N^{\dagger} N\right) S^{*} \Delta\left(T^{k}\right)^{-1} \widetilde{T} N N^{\dagger}
\end{array}\right] U^{*},
$$

where $\Delta$ is defined as in Theorem 3.9.

Proof. We observe that $A^{c, \dagger}=A^{\dagger} A A^{d, \dagger}$. Now, (24) follows by a simple computation from (21) and (23).

The following result provides a necessary and sufficient condition for a matrix to be $k$-DMP by using the core EP decomposition. As a consequence, it follows that the class $\mathbb{C}_{n}^{k, \dagger}$ is a subset of $\mathbb{C}_{n}^{k, d \dagger}$. Theorem 3.13. Let $A \in \mathbb{C}^{n \times n}$ be a matrix of index $k$ written as in (6). Then $A \in \mathbb{C}_{n}^{k, d \dagger}$ if and only if $\mathcal{N}\left(N^{*}\right) \subseteq \mathcal{N}(\widetilde{T})$.

Proof. We suppose that $A \in \mathbb{C}_{n}^{k, d \dagger}$. By using (15) and (21) it is easy to see that $A^{d, \dagger} A^{k}=A^{k} A^{d, \dagger}$ if and only if $\widetilde{T}\left(I_{n-t}-N N^{\dagger}\right)=0$, which is equivalent to $\mathcal{N}\left(N^{*}\right) \subseteq \mathcal{N}(\widetilde{T})$. 
Now, we establish another characterization for $k$-DMP matrices.

Theorem 3.14. Let $A \in \mathbb{C}^{n \times n}$ be a matrix of index $k$. Then the following statements are equivalent:

(a) $A$ is a $k$-DMP matrix;

(b) $A^{d, \dagger}=A^{d}$;

(c) $A^{c, \dagger}=A^{\dagger, d}$.

Proof. $(a) \Longleftrightarrow(b)$ From (14) and (21) we have that $A^{d, \dagger}=A^{d}$ if and only if $\widetilde{T}\left(I_{n-t}-N N^{\dagger}\right)=0$, which is equivalent to the fact that $A$ is a $k$-DMP matrix by Theorem 3.13.

$(b) \Rightarrow(c)$ Suppose that $A^{d, \dagger}=A^{d}$ holds. Then $A^{c, \dagger}=A^{\dagger} A\left(A^{d} A A^{\dagger}\right)=A^{\dagger} A A^{d, \dagger}=A^{\dagger} A A^{d}=A^{\dagger, d}$.

$(c) \Rightarrow(b)$ Assume that $A^{c, \dagger}=A^{\dagger, d}$ is true. By Corollary $3.3(a)$ we obtain $\mathcal{N}\left(A^{k} A^{\dagger}\right)=\mathcal{N}\left(A^{k}\right)$. According to Theorem $3.2(\mathrm{~b})$ and (9) we have $A^{d, \dagger}=A_{\mathcal{R}\left(A^{k}\right), \mathcal{N}\left(A^{k}\right)}^{(2)}=A^{d}$.

Remark 3.15. The class $\mathbb{C}_{n}^{k, \dagger}$ is a proper subset of $\mathbb{C}_{n}^{k, d \dagger}$. For example, if we take

$$
A=\left[\begin{array}{rrrr}
1 & 0 & 1 & -1 \\
0 & 1 & 1 & -1 \\
0 & 0 & 0 & 1 \\
0 & 0 & 0 & 0
\end{array}\right]
$$

we have that $\operatorname{Ind}(A)=2$,

$$
A^{\dagger}=\left[\begin{array}{rrcc}
2 / 3 & -1 / 3 & 1 / 3 & 0 \\
-1 / 3 & 2 / 3 & 1 / 3 & 0 \\
1 / 3 & 1 / 3 & 2 / 3 & 0 \\
0 & 0 & 1 & 0
\end{array}\right] \quad \text { and } \quad A^{d}=\left[\begin{array}{cccc}
1 & 0 & 1 & 0 \\
0 & 1 & 1 & 0 \\
0 & 0 & 0 & 0 \\
0 & 0 & 0 & 0
\end{array}\right] \text {. }
$$

It is easy to see that $A^{2} A^{d, \dagger}=A^{d, \dagger} A^{2}$, but $A^{2} A^{\dagger} \neq A^{\dagger} A^{2}$.

It can be derived from the following result that the class $\mathbb{C}_{n}^{k, \dagger}$ is a subset of $\mathbb{C}_{n}^{k, \dagger d}$.

Theorem 3.16. Let $A \in \mathbb{C}^{n \times n}$ be a matrix of index $k$ written as in (6). Then $A \in \mathbb{C}_{n}^{k, \dagger d}$ if and only if $\mathcal{N}(N) \subseteq \mathcal{N}(S)$.

Proof. Assume that $A \in \mathbb{C}_{n}^{k, \dagger d}$ is satisfied. By using (15) and (22) it is easy to see that $A^{\dagger, d} A^{k}=A^{k} A^{\dagger, d}$ if and only if the following conditions simultaneously hold:

(i) $T^{*} \Delta T^{k}=T^{k} T^{*} \Delta+\widetilde{T}\left(I_{n-t}-N^{\dagger} N\right) S^{*} \Delta$, 
(ii) $\left(I_{n-t}-N^{\dagger} N\right) S^{*} \Delta T^{k}=0$,

(iii) $T^{*} \Delta \widetilde{T}=T^{k} T^{*} \Delta T^{-k} \widetilde{T}+\widetilde{T}\left(I_{n-t}-N^{\dagger} N\right) S^{*} \Delta T^{-k} \widetilde{T}$,

(iv) $\left(I_{n-t}-N^{\dagger} N\right) S^{*} \Delta \widetilde{T}=0$.

Hence, (ii) implies that $S\left(I_{n-t}-N^{\dagger} N\right)=0$ since $T$ and $\Delta$ are nonsingular. So, $\mathcal{N}(N) \subseteq \mathcal{N}(S)$.

Conversely, we have $S\left(I_{n-t}-N^{\dagger} N\right)=0$. Now, it is easy to check that conditions (i)-(iv) are valid.

Our next result establishes another characterization for dual $k$-DMP matrices.

Theorem 3.17. Let $A \in \mathbb{C}^{n \times n}$ be a matrix of index $k$. Then the following statements are equivalent:

(a) A is a dual k-DMP matrix;

(b) $A^{\dagger, d}=A^{d}$;

(c) $A^{c, \dagger}=A^{d, \dagger}$.

Proof. $(a) \Rightarrow(b)$ Assume that $A$ is a dual $k$-DMP matrix. By Theorem 3.16, $S\left(I_{n-t}-N^{\dagger} N\right)=0$. Therefore,

$$
A^{\dagger}=U\left[\begin{array}{cc}
T^{-1} & -T^{-1} S N^{\dagger} \\
0 & N^{\dagger}
\end{array}\right]
$$

by (19). Now, from (14) we obtain $A^{\dagger, d}=A^{d}$.

$(b) \Rightarrow(a)$ Let $A^{\dagger} \in \mathbb{C}^{n \times n}$ be written as in (19). Since $A^{\dagger, d}=A^{d}$, from (14) and (22) we get

$$
U\left[\begin{array}{cc}
T^{*} \Delta & T^{*} \Delta T^{-k} \widetilde{T} \\
\left(I_{n-t}-N^{\dagger} N\right) S^{*} \Delta & \left(I_{n-t}-N^{\dagger} N\right) S^{*} \Delta T^{-k} \widetilde{T}
\end{array}\right] U^{*}=U\left[\begin{array}{cc}
T^{-1} & \left(T^{k+1}\right)^{-1} \widetilde{T} \\
0 & 0
\end{array}\right] U^{*} .
$$

Hence $\left(I_{n-t}-N^{\dagger} N\right) S^{*}=0$, since $\Delta$ is nonsigular. Therefore $S\left(I_{n-t}-N^{\dagger} N\right)=0$ and Theorem 3.16 completes the proof.

$(b) \Rightarrow(c)$ Since $A^{\dagger, d}=A^{d}$, we get $A^{c, \dagger}=\left(A^{\dagger} A A^{d}\right) A A^{\dagger}=A^{\dagger, d} A A^{\dagger}=A^{d} A A^{\dagger}=A^{d, \dagger}$.

$(c) \Rightarrow(b)$ Assume that $A^{c, \dagger}=A^{d, \dagger}$ holds. By Corollary $3.3(b)$ we obtain $\mathcal{R}\left(A^{\dagger} A^{k}\right)=\mathcal{R}\left(A^{k}\right)$. According to Theorem $3.2(\mathrm{~d})$ and (9) we have $A^{\dagger, d}=A_{\mathcal{R}\left(A^{k}\right), \mathcal{N}\left(A^{k}\right)}^{(2)}=A^{d}$.

In [7, Theorems 3.3, 3.5, and 3.6], it was proved that $A$ is $k$-EP if and only if $A^{c, \dagger}=A^{d}$ if and only if $A^{d, \dagger}=A^{\dagger, d}$. Moreover, if $A$ is a $k$-EP matrix, by Theorems 3.10 to 3.17 we have that $A$ is a dual $k$-DMP matrix and, moreover, $A^{\dagger, d}=A^{d}$ and $A^{c, \dagger}=A^{d, \dagger}$. Thus, we have the following result.

Corollary 3.18. Let $A \in \mathbb{C}^{n \times n}$ be a matrix of index $k$. Then $A$ is $k$-EP matrix if and only if $A^{c, \dagger}=A^{d, \dagger}=A^{\dagger, d}=A^{d}$. 
Remark 3.19. The class $\mathbb{C}_{n}^{k, \dagger}$ is a proper subset of $\mathbb{C}_{n}^{k, \dagger d}$. For example, if we take

$$
A=\left[\begin{array}{rrrr}
1 & 0 & 1 & 2 \\
0 & 1 & 1 & 2 \\
0 & 0 & 2 & 4 \\
0 & 0 & -1 & -2
\end{array}\right]
$$

we have that $\operatorname{Ind}(A)=2$,

$$
A^{\dagger}=\left[\begin{array}{cccc}
1 & 0 & -2 / 5 & 1 / 5 \\
0 & 1 & -2 / 5 & 1 / 5 \\
0 & 0 & 2 / 25 & -1 / 25 \\
0 & 0 & 4 / 25 & -2 / 25
\end{array}\right], \quad \text { and } \quad A^{d}=\left[\begin{array}{cccc}
1 & 0 & 1 & 2 \\
0 & 1 & 1 & 2 \\
0 & 0 & 0 & 0 \\
0 & 0 & 0 & 0
\end{array}\right] \text {. }
$$

It is easy to see that $A^{2} A^{\dagger, d}=A^{\dagger, d} A^{2}$, but $A^{2} A^{\dagger} \neq A^{\dagger} A^{2}$.

The following interesting result is a characterization for $k$-EP matrices and it can be easily derived from Theorems 3.10, 3.13, and 3.16.

Theorem 3.20. $\mathbb{C}_{n}^{k, \dagger}=\mathbb{C}_{n}^{k, d \dagger} \cap \mathbb{C}_{n}^{k, \dagger d}$.

Now, we give another characterization for $k$-EP matrices by using the CMP inverse.

Theorem 3.21. The classes of matrices $k-E P$ and $k$-CMP are coincide.

Proof. Since $A^{c, \dagger}=Q_{A} A^{d} P_{A}$, we observe that $A^{k} Q_{A}=P_{A} A^{k}=A^{k}$. Then, $A^{k} A^{c, \dagger}=A^{k} Q_{A} A^{d} P_{A}=$ $A^{k} A^{d} A A^{\dagger}=A^{k+1} A^{d} A^{\dagger}=A^{k} A^{\dagger}$. Also, we have $A^{c, \dagger} A^{k}=Q_{A} A^{d} P_{A} A^{k}=A^{\dagger} A A^{d} A^{k}=A^{\dagger} A^{k+1} A^{d}=$ $A^{\dagger} A^{k}$. Therefore, $A^{k} A^{c, \dagger}=A^{c, \dagger} A^{k}$ if and only if $A^{k} A^{\dagger}=A^{\dagger} A^{k}$, i.e., $\mathbb{C}_{n}^{k, \dagger}=\mathbb{C}_{n}^{k, c \dagger}$.

\section{New characterizations for core EP inverses and extensions}

According to [9], it is well known that $X=A^{\oplus}$ is equivalent to $A X A=A, A X^{2}=X$, and $(A X)^{*}=$ $A X$. By exploiting the condition $A X^{2}=X$, we shall obtain a new necessary and sufficient condition for a matrix to be the core EP inverse. Moreover, motivated by [8, Lemma 3.3] we prove that the core EP inverse of a square matrix can be characterized by two new sets of three equations each one. Before that, we present two auxiliary lemmas.

Lemma 4.1. Let $A, X \in \mathbb{C}^{n \times n}$ with $\operatorname{Ind}(A)=k$ such that $A X^{2}=X$. Then $\mathcal{R}(X) \subseteq \mathcal{R}\left(A^{k}\right)$ (and, consequently, $\mathcal{N}\left(\left(A^{k}\right)^{*}\right) \subseteq \mathcal{N}\left(X^{*}\right)$ ). If, in addition, $X A^{k+1}=A^{k}$ then $\mathcal{R}(X)=\mathcal{R}\left(A^{k}\right)$ (and, consequently, $\mathcal{N}\left(X^{*}\right)=\mathcal{N}\left(\left(A^{k}\right)^{*}\right)$ and $X$ is a $\{2\}$-inverse of $A$. 
Proof. Since $A X^{2}=X$, by induction it then follows that

$$
X=A X^{2}=A X X=A\left(A X^{2}\right) X=A^{2} X X^{2}=A^{2}\left(A X^{2}\right) X^{2}=\cdots=A^{k}\left(A X^{2}\right) X^{k} .
$$

Thus, $\mathcal{R}(X) \subseteq \mathcal{R}\left(A^{k}\right)$ and then $\mathcal{N}\left(\left(A^{k}\right)^{*}\right) \subseteq \mathcal{N}\left(X^{*}\right)$.

Now, we assume that $X A^{k+1}=A^{k}$ is also fulfilled. It is clear that now $\mathcal{R}(X)=\mathcal{R}\left(A^{k}\right)$ and then $\mathcal{N}\left(\left(A^{k}\right)^{*}\right)=\mathcal{N}\left(X^{*}\right)$. On the other hand, since $\mathcal{R}(X) \subseteq \mathcal{R}\left(A^{k}\right)$ can be equivalently expressed as $P_{A^{k}} X=X$, we conclude that $X A X=X A P_{A^{k}} X=X A^{k+1}\left(A^{k}\right)^{\dagger} X=P_{A^{k}} X=X$.

In the following lemma, we prove that the equation $X A^{k+1}=A^{k}$ in (2) is redundant and then it can be dropped out as we establish in Theorem 4.3.

Lemma 4.2. Let $A \in \mathbb{C}^{n \times n}$ with $\operatorname{Ind}(A)=k$. Then the following statements are equivalent:

(a) $X A^{k+1}=A^{k}$ and $\mathcal{R}(X) \subseteq \mathcal{R}\left(A^{k}\right)$;

(b) $X A X=X$ and $\mathcal{R}(X)=\mathcal{R}\left(A^{k}\right)$.

Proof. $(a) \Rightarrow(b)$ It is a direct consequence from the proof of Lemma 4.1.

$(b) \Rightarrow(a)$ Notice that $\mathcal{R}\left(A^{k}\right) \subseteq \mathcal{R}(X)$ can be equivalently expressed as $P_{X} A^{k}=A^{k}$. Postmultiplying the equation $X A X=X$ on the right-hand by $X^{\dagger} A^{k}$ leads to $X A P_{X} A^{k}=P_{X} A^{k}$. So, $X A^{k+1}=A^{k}$.

Theorem 4.3. Let $A, X \in \mathbb{C}^{n \times n}$ with $\operatorname{Ind}(A)=k$. Then the following statements are equivalent:

(a) $X$ is the core $E P$ of $A$;

(b) $X A^{k+1}=A^{k}, A X^{2}=X$, and $(A X)^{*}=A X$;

(c) $X A X=X,(A X)^{*}=A X$, and $\mathcal{R}(X)=\mathcal{R}\left(A^{k}\right)$.

Proof. $(a) \Rightarrow(b)$ We suppose that $X$ is the core EP of $A$. From [10, Theorem 2.2 and 3.2], it is not hard to see that $A X^{2}=X$. Therefore (b) holds from (2).

$(b) \Rightarrow(a)$ We assume that $(b)$ is true. By Lemma 4.1 we have $X A X=X$ and $\mathcal{R}(X)=\mathcal{R}\left(A^{k}\right)$. Thus,

(2) leads to $(a)$.

$(a) \Longleftrightarrow(c)$ The proof follows as a direct application of (2) and Lemma 4.2.

We close this paper by providing some extensions valid for $k$-core EP matrices. In [2, Theorem 3$]$ the following equivalences were proved for at most index 1 matrices:

$$
A \text { is } \mathrm{EP} \quad \Longleftrightarrow \quad A A^{\oplus}=A^{\oplus} A \quad \Longleftrightarrow \quad A^{\oplus}=A^{\#} .
$$

We will give a generalization of this assertion for $k$-core EP matrices. 
Theorem 4.4. Let $A \in \mathbb{C}^{n \times n}$ be a matrix of index $k$ written as in (6). Then the following statements are equivalent:

(a) A is a k-core EP matrix;

(b) $\widetilde{T}=0$;

(c) $A^{\oplus}=A^{d}$.

Moreover, in this case, $A$ is a $k$-DMP matrix and $A^{d, \dagger}=A^{\oplus}=A^{d}$.

Proof. Let $A \in \mathbb{C}^{n \times n}$ be written as in (6). By (13)-(15) it is obvious that $A$ is a $k$-core EP matrix, i.e., $A^{\oplus} A^{k}=A^{k} A^{\oplus}$, if and only if $\widetilde{T}=0$ if and only if $A^{\oplus}=A^{d}$.

Now, item (b) implies $\widetilde{T}\left(I_{n-t}-N N^{\dagger}\right)=0$. Next, Theorem 3.13 implies that $A$ is a $k$-DMP matrix. Finally, from (13), (14) and (21) we have $A^{d, \dagger}=A^{\oplus}=A^{d}$.

We observe that if $\operatorname{Ind}(A) \leq 1$, then $A^{\oplus}=A^{\oplus}$ and $A^{d}=A^{\#}$, and in consequence the above theorem generalizes the result in (25). Moreover, Theorem 4.4 describes the inclusion of the class $\mathbb{C}_{n}^{k, \oplus}$ into the class $\mathbb{C}_{n}^{k, d \dagger}$ for $k>1$.

Remark 4.5. Notice that the class $\mathbb{C}_{n}^{k, \oplus}$ is a proper subset of $\mathbb{C}_{n}^{k, d \dagger}$. For example, if we take

$$
A=\left[\begin{array}{rrrr}
1 & -1 & 1 & 0 \\
0 & 0 & 0 & 0 \\
0 & 1 & 0 & 0 \\
0 & 0 & 1 & 0
\end{array}\right]
$$

we have that $\operatorname{Ind}(A)=3$,

$$
A^{\dagger}=\left[\begin{array}{rrrr}
1 & 0 & 1 & -1 \\
0 & 0 & 1 & 0 \\
0 & 0 & 0 & 1 \\
0 & 0 & 0 & 0
\end{array}\right], \quad A^{d}=\left[\begin{array}{cccc}
1 & 0 & 1 & 0 \\
0 & 0 & 0 & 0 \\
0 & 0 & 0 & 0 \\
0 & 0 & 0 & 0
\end{array}\right] \quad \text { and } \quad A^{\oplus}=\left[\begin{array}{cccc}
1 & 0 & 0 & 0 \\
0 & 0 & 0 & 0 \\
0 & 0 & 0 & 0 \\
0 & 0 & 0 & 0
\end{array}\right] .
$$

It is easy to see that $A^{3} A^{d, \dagger}=A^{d, \dagger} A^{3}$, but $A^{3} A^{\oplus} \neq A^{\oplus} A^{3}$.

\section{Acknowledgement}

The authors would like to thank the referees for their valuable comments which helped to improve the readability of the manuscript. 


\section{References}

[1] A. Ben-Israel, T.N.E Greville, Generalized Inverses: Theory and Applications, second ed., Springer-Verlag, New York, 2003.

[2] O.M. Baksalary, G. Trenkler, Core inverse of matrices, Linear and Multilinear Algebra, 58 (6) (2010) 681-697.

[3] C.Y. Deng, H.K. Du, Representations of the Moore-Penrose inverse of $2 \times 2$ block operator valued matrices, J. Korean Math. Soc. 46 (2009) 1139-1150.

[4] D.E. Ferreyra, F. Levis, N. Thome, Revisiting of the core EP inverse and its extension to rectangular matrices, Quaestiones Mathematicae, 41 (2) (2018) 265-281.

[5] S. Malik, N. Thome, On a new generalized inverse for matrices of an arbitrary index, Applied Mathematics and Computation, 226 (2014) 575-580.

[6] S. Malik, L. Rueda, N. Thome, The class of m-EP and m-normal matrices, Linear and Multilinear Algebra, 64 (11) (2016) 2119-2132.

[7] M. Mehdipour, A. Salemi, On a new generalized inverse of matrices, Linear and Multilinear Algebra, 66 (5) (2018) 1046-1053.

[8] K.M. Prasad, K.S. Mohana, Core EP inverse, Linear and Multilinear Algebra, 62 (3) (2014) $792-802$.

[9] X. Wang, X. Liu Characterizations of the core inverse and the core partial ordering, Linear and Multilinear Algebra, 63 (9) (2014) 1829-1836.

[10] X. Wang, Core-EP decomposition and its applications, Linear Algebra and its Applications, 508 (2016) 289-300.

[11] D.S. Rakić, N.C. Dincić, D.S. Djordjević, Core inverse and core partial order of Hilbert space operators, Applied Mathematics and Computation, 244 (2014) 283-302.

[12] V. Rakoćević, Y. Wei, The representation and approximation of the W-weighted Drazin inverse of linear operators in Hilbert space, Applied Mathematics and Computation, 141 (2003) 455-470.

[13] C.R. Rao, S.K. Mitra, Generalized inverse of matrices and its Applications, John Wiley and Sons, New York, 1971. 
[14] P.S. Stanimirović, N. Katsikis, H. Ma, Representations and properties of the $W$-Weighted Drazin inverse, Linear Algebra and its Applications, 65 (6) (2017) 1080-1096.

[15] H. Wang, J. Chen, Weak group inverse, http://arxiv.org/abs/1704.08403v1

[16] Y. Wei, A characterization and representation of the generalized inverse $A_{T, S}^{(2)}$ and its applications, Linear Algebra and its Applications, 280 (2-3) (1998) 87-96. 